\title{
Design of Tunable Monopole Arm Planar Spiral Antenna for Cognitive Radio
}

\author{
Rahul Yadav \\ Department of Electronics and Telecommunication, University of Mumbai, Santacruz East, Mumbai 400032, India \\ Correspondence should be addressed to Rahul Yadav; ryrahulyadav01@gmail.com
}

Received 23 April 2014; Revised 4 July 2014; Accepted 9 July 2014; Published 24 July 2014

Academic Editor: Sarod Yatawatta

Copyright ( 2014 Rahul Yadav. This is an open access article distributed under the Creative Commons Attribution License, which permits unrestricted use, distribution, and reproduction in any medium, provided the original work is properly cited.

\begin{abstract}
This paper presents a spiral antenna design operating in the frequency range of 1-15 GHz having both selective notch bands and wideband response. The main feed arm of spiral antenna is configured as rectangular monopole of width quarter wavelength to achieve impedance matching with standard $50 \Omega$ excitation. Frequency tuning in the design is achieved by placing varactor diode at an appropriate position along the spiral arms and in the ground plane. The design offers a peak gain of $3.4 \mathrm{~dB}$ (simulated) and $3 \mathrm{~dB}$ (measured). The unique frequency response of antenna makes its suitable to be used for front-end system of cognitive radio for sensing the spectrum in various modes.
\end{abstract}

\section{Introduction}

The development of antennas in the past five decades has gone through various design changes, in order to enhance the overall radiation characteristics. The antennas which are being used in the present wireless communication technology are either having a multiple frequency response or a wideband response. But the rapid growth in the field of microwave communication has put a demand to develop antenna with wideband response due to their high data rates, great capacity, simplex design, and low power consumption. However, an antenna with ultra-wide band response mainly finds its application in electronic warfare and military purpose. It is found in [1-3] that, frequency independent antennas like logspiral, rectangular spiral and Archimedean-spiral are suitable to provide a wideband frequency response, but the problem that arises in such antennas is with the feeding system due to increased antenna impedance which is generally in the range of (140-200) $\Omega$. So special wideband balun is required to be designed [4-6] for providing an impedance matching in the wide microwave range of frequency thereby making the whole system much more bulky and putting a constraint in their application.

Another main drawback of UWB antennas is their interference with existing communication channels like GSM
(900-1800 GHz), UMTS (1.92-2.71 GHz), local area network (WLAN $5.15-5.825 \mathrm{GHz}$ ), and worldwide interoperability for microwave access (Wi-MAX, 3.3-3.7 GHz) IEEE 802.11a in the United States $(5.15-5.35 \mathrm{GHz}, 5.725-5.825 \mathrm{GHz})$. The interference produces a high impulse noise which is difficult to eliminate at the receiver system and thereby increases the complexity of whole communication system.

The cognitive radio system is the upcoming wireless technology for very high data rates even beyond $100 \mathrm{Mbps}$. Such a system requires a few design specifications which the antenna should meet. For example, antennas are expected to have a dual nature of frequency response, that is, wideband response to support higher data rates and multiple frequency bands so that they can support present wireless technology and the existing applications. Also features like frequency response conversion from wideband to multiple band and vice-versa and coarse and fine tuning for lower frequency range to support variety of application will make the cognitive system more efficient and smarter and allow to sense the spectrum in various modes like full mode (wideband spectrum scanning) and licenced and unlicensed band mode (scanning only selective frequency bands). This will certainly help to tackle the problem of spectrum scarcity.

In this paper, a design of spiral antenna is presented to meet the design requirements of upcoming and present 
wireless technology. The spiral antenna is designed to operate in the frequency range of $1-15 \mathrm{GHz}$. The arms of spiral are configured as rectangular monopole to provide an impedance matching with standard $50 \Omega$ excitation. A frequency response switching between selective band and wideband along with the tuning is achieved by changing the antenna electrical length with the placement of varactor diode at an appropriate position along the spiral arms. Optimization of ground plane is also performed to investigate its effect on the frequency response.

\section{Spiral Antenna Design}

The antenna is designed by taking a FR-4 substrate of permittivity $\left(\varepsilon_{r}=4.3\right)$ and loss tangent $(\delta=0.025)$. The procedure begins by configuring each arm of the spiral as rectangular monopole. The width of $\operatorname{arm}(w)$ is taken as $\lambda_{g} / 4$, where

$$
\begin{gathered}
\lambda_{g}=\frac{\lambda_{r}}{\sqrt{\varepsilon_{\mathrm{eff}}}}=\frac{c}{f_{r} \sqrt{\varepsilon_{\mathrm{eff}}}}, \\
\varepsilon_{\mathrm{eff}}=\frac{\varepsilon_{r}+1}{2},
\end{gathered}
$$

where $\varepsilon_{\text {eff }}$ is effective dielectric constant, $f_{r}$ is higher resonant frequency, and $\lambda_{r}$ is resonant wavelength.

Therefore, assuming $9.2 \mathrm{GHz}$ as the higher resonant frequency, the width $(w)$ is calculated as $5 \mathrm{~mm}$. Figure 1(a) shows the basic geometrical design of spiral antenna which is composed of six arms. The alternate arms width has been kept as $2 w$, so as to increase the overall effective area which will eventually help to shift frequency bands response towards lower range also. Since the presented design is configured as microstrip, the ground plane dimensions are calculated using transmission line model [7]. The calculated ground plane dimension is at length $\left(L_{g}\right)$ of $157 \mathrm{~mm}$ and width $\left(W_{g}\right)$ of $126 \mathrm{~mm}$. Figure 1(b) shows the layout of modified ground plane having a rectangular slot of cut-out width $2 \mathrm{~mm}$ along with the connection pad for varactor diodes which are incorporated for frequency tuning later.

2.1. Frequency Tuning and Biasing. The coarse and fine tuning of frequency response in the designed spiral antenna is achieved by placing varactor diode at an appropriate position along the spiral arm and ground plane. Figure 2 shows the placement of varactor diodes along with $\mathrm{RF} / \mathrm{DC}$ isolation network. Varactor diode model MA46H071 GaAs is used in the design. The main purpose for introducing varactor diode instead of PIN diode is to control the antenna electrical length $(\beta l)$ and hence the resonant frequency with the help of DC bias. The equivalent circuit of diode proposed by the vendor is a series of RLC circuit with a parallel capacitance as shown in Figure 5(a). For diode model, the typical value of a series of resistance $\left(R_{s}\right)$ is $5 \Omega$ and a series of inductance $\left(L_{s}\right)$ is $5 \mathrm{nH}$. The plot for junction capacitance $\left(C_{j}\right)$ as a function of reverse bias voltage for MA46H071 is shown in Figure 5(b).

The DC isolation in the design is given by a capacitor which offers low impedance at the RF frequency. So a DC blocking capacitor of $220 \mathrm{pF}$ is placed along the main arm of spiral as shown in Figure 2(a).

It is important to note that for the placement of diode and DC blocking capacitor, a gap of $1 \mathrm{~mm}$ has been formed at the respective arm. This gap discontinuity introduces an extra capacitance in the form of equivalent $\prod$-network as shown in Figure 3. However, the extra capacitance $\left(C_{x}\right)$ given by reversed biased varactor diode and DC block will modify the equivalent circuit as shown in Figure 4 . The numerical value of the capacitances can be found [8] using (2):

$$
\begin{gathered}
C_{p}=0.5 C_{e}, \\
C_{g}=0.5 C_{o}-0.25 C_{e} \\
\frac{C_{o}}{W} \mathrm{pF} / \mathrm{m}=\left(\frac{\varepsilon_{r}}{9.6}\right)^{0.8}\left(\frac{s}{W}\right)^{m o} \exp (k o), \\
\frac{C_{e}}{W} \mathrm{pF} / \mathrm{m}=12\left(\frac{\varepsilon_{r}}{9.6}\right)^{0.9}\left(\frac{s}{W}\right)^{m e} \exp (k e),
\end{gathered}
$$

where mo $=(W / h)[0.619 \log (W / h)-0.3853], k o=4.26-$ $1.453 \log (W / h)$, for $0.1 \leq s / W \leq 1.0$, me $=0.8675$, $k e=$ $2.043(W / h)^{0.12}$, for $0.1 \leq s / W \leq 0.3$. With $s=1 \mathrm{~mm}, W=$ $5 \mathrm{~mm}$ for arm 1 and $10 \mathrm{~mm}$ for arm $4, \varepsilon_{r}=4.3, h=1.6 \mathrm{~mm}$.

Next, the radio frequency (RF) signal is isolated by putting a low pass filter (LPF) between the +ve connection pad and diode cathode terminal via a high impedance line $(100 \Omega)$. The LPF is a ceramic SMD component of Johanson filter. Figure 6 shows the plot of RF isolation, which is well below $-30 \mathrm{~dB}$ indicating that RF signal will be isolated effectively. It is important to note that at higher frequencies, parasitic package effect of lumped components becomes more significant; here in the design this has been accounted to some extent by the use of high impedance line along with the filter which will provide an impedance mismatch between the antenna and DC source and will isolate RF.

To achieve frequency tuning at a greater extent, the ground plane of antenna is modified by introducing a square slot and three varactor diodes are placed between the slots in three different directions as shown in Figure 2(b). Lastly, the antenna frequency tuning network is biased via a typical voltage regulator circuit made using IC-LM317 as shown in Figure 7.

\section{Results and Discussion}

A numerical analysis of the proposed spiral antenna design is done using computer simulation tool (CST-2010) which works on finite integral numerical technique (FIT) [9]. Initially the design is solved without any diode biasing and a unique frequency response having both selective notches and wideband in the range of $1-15 \mathrm{GHz}$ is achieved as shown in Figure 8(a). The excited wideband response is due to the increase in adjacent spiral arm width which allows a better continuous exponential decay $\left(e^{-\beta l}\right)$ of antenna surface current from the main arm towards its progressive arms. On the other hand, multiple notch frequency bands are resonated towards the lower side of spectrum (L-S band) due to antenna 




(a)

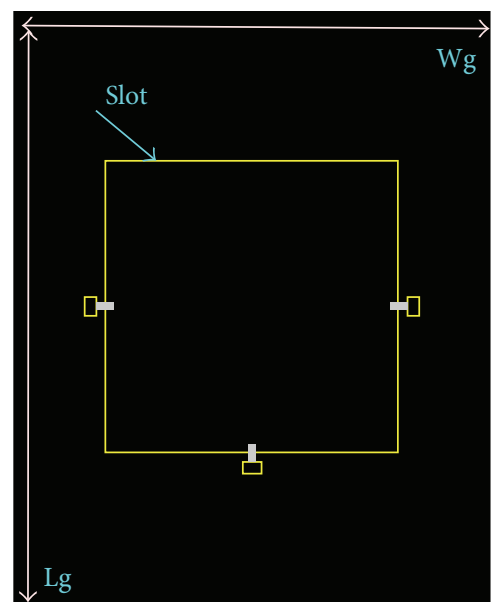

(b)

FIGURE 1: Spiral antenna layout (top view), (b) antenna modified ground plane.

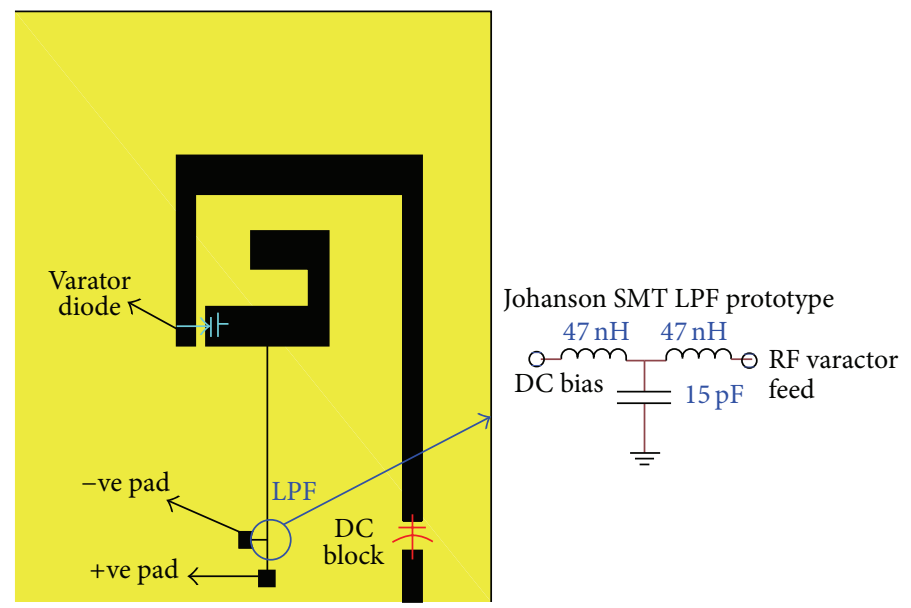

(a)

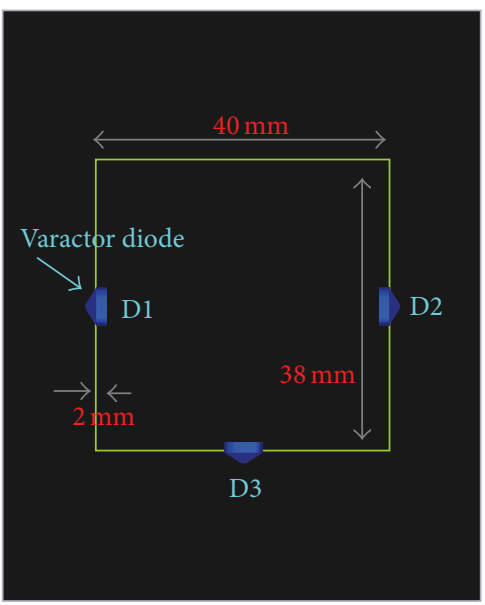

(b)

FIGURE 2: (a) Spiral with diode and RF/DC isolation, (b) varactor diode placement in ground plane.
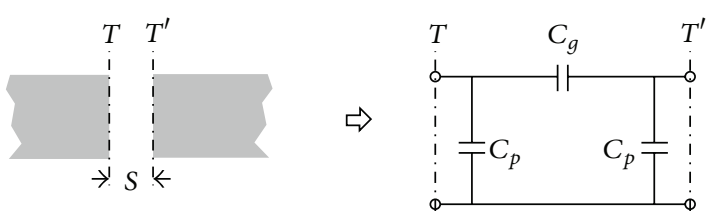

FIGURE 3: Gap discontinuity equivalent circuit.

overall effective length and discontinuities between the adjacent arms which creates multiple maxima and minima in the current distribution at the rejection frequencies. A close agreement in the simulated and measured result of reflection coefficient can be seen in Figure 8(a).

The comparative wideband gain plot of simulated and measured result for the design without any diode biasing is correspondingly shown in Figure 8(b). The design offers a peak simulated gain of $3.4 \mathrm{~dB}$ and measured gain of $3.25 \mathrm{~dB}$ in the full band of $1 \mathrm{GHz}$ to $15 \mathrm{GHz}$. A detail frequency

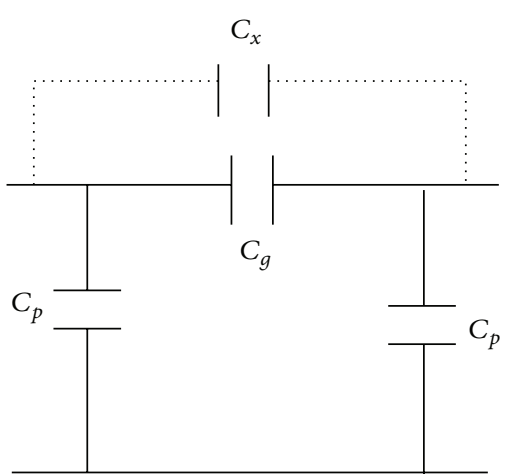

Figure 4: Modified equivalent circuit.

and gain analysis are illustrated in Table 1 . The surface current distribution simulated for the notch frequency bands at $1.93 \mathrm{GHz}, 2.79 \mathrm{GHz}, 3.47 \mathrm{GHz}$, and $4.45 \mathrm{GHz}$ is shown in Figure 9. It is observed that since the antenna surface current is relatively the same, the E-field pattern produced is 


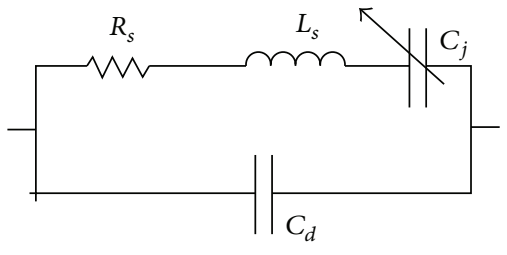

(a)

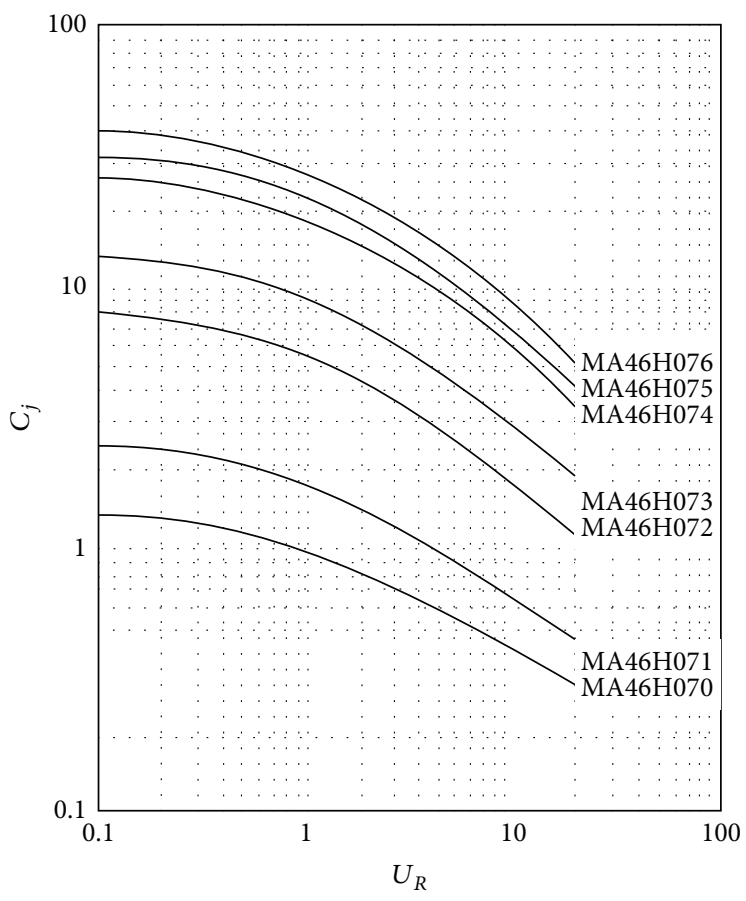

(b)

FIgURE 5: (a) Equivalent circuit model of varactor diode, (b) plot of diode junction capacitance.

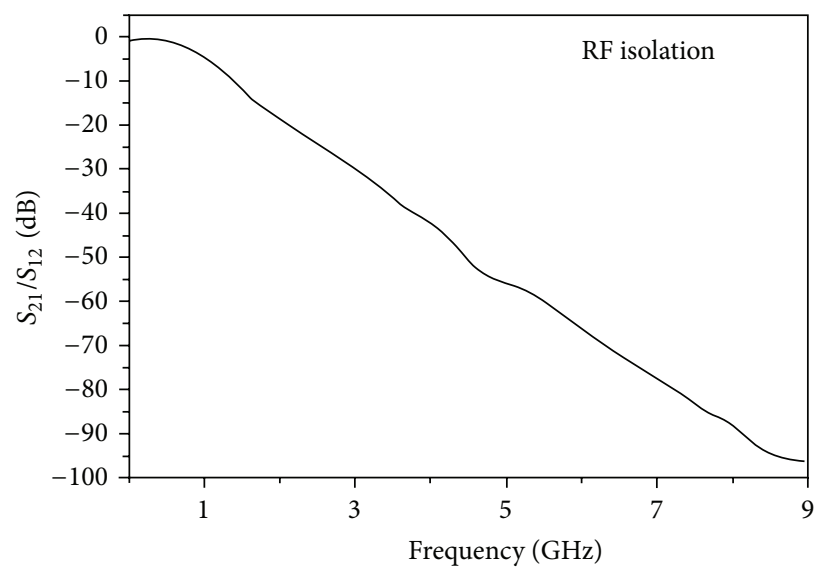

FIGURE 6: Plot of RF isolation versus frequency.

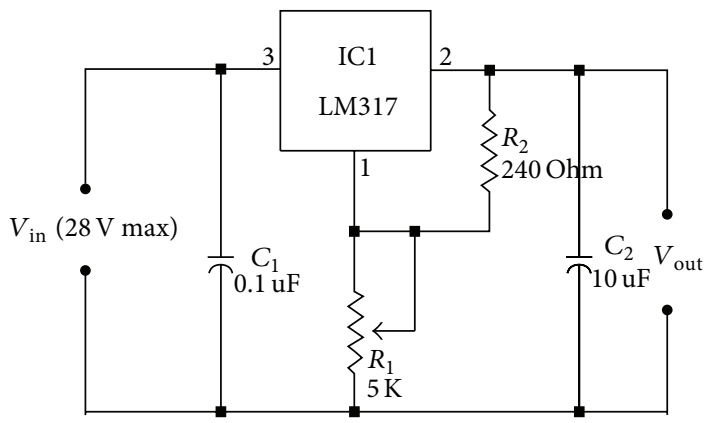

FIgURE 7: Typical voltage regulator circuit. 




(a)

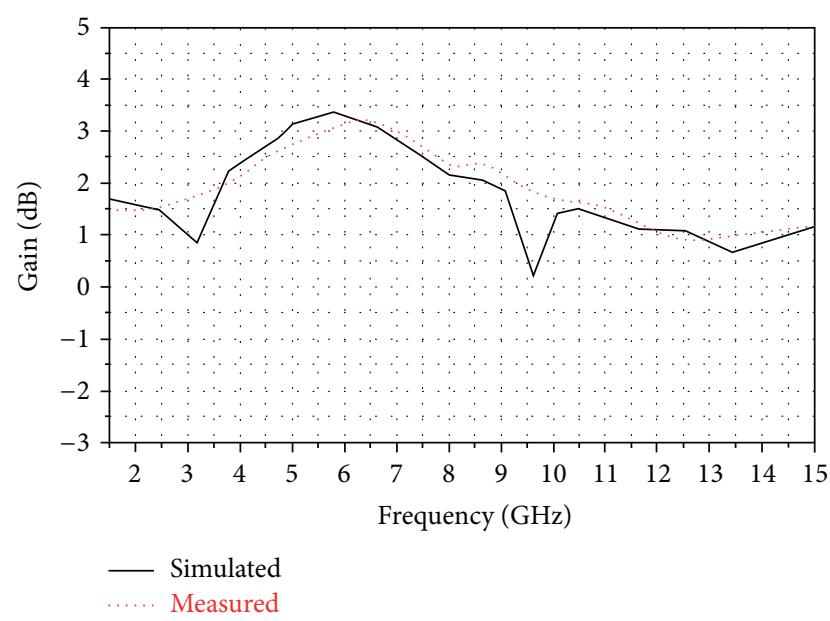

(b)

FIGURE 8: Plot of (a) reflection coefficient, (b) gain (without diode).

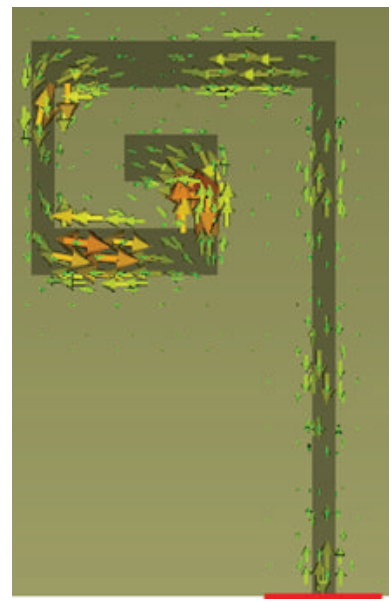

(a)

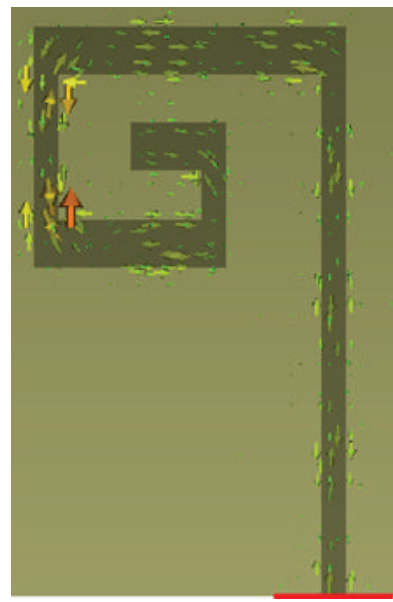

(b)



(c)

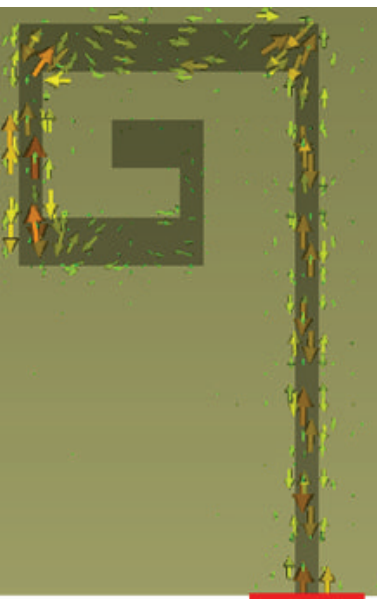

(d)

Figure 9: Antenna surface current at (a) $1.93 \mathrm{GHz}$, (b) $2.79 \mathrm{GHz}$, (c) $3.47 \mathrm{GHz}$, and (d) $4.45 \mathrm{GHz}$.

TABLE 1: Frequency band and gain analysis for antenna with diodes OFF.

\begin{tabular}{lcc}
\hline Parameter & $\begin{array}{c}\text { Simulated } \\
\text { frequency }(\mathrm{GHz})\end{array}$ & $\begin{array}{c}\text { Peak gain }(\mathrm{dB}) \\
\text { Simulated/measured }\end{array}$ \\
\hline $\begin{array}{l}\text { Selective } \\
\text { resonant bands }\end{array}$ & $1.93,2.79,3.47,4.45$ & $2.78 / 2.65$ \\
Wideband & $5.7-15$ & $3.4 / 3$ \\
\hline
\end{tabular}

comparable as shown in Figure 10. The antenna under no bias condition has vertical polarization all the selective resonant frequencies shown in Table 1.

Next, in order for the antenna to support multiple frequency channels, there is a need to convert wideband into notch bands. This is achieved by placing a varactor between the 3rd and 4th arm of the spiral as discussed in Section 2, which suitably helps to vary the antenna electrical length and eliminate the continuous time decay current component. This
TABLE 2: Resonated frequencies at different diode junction capacitance.

\begin{tabular}{lcc}
\hline $\begin{array}{l}\text { Bias } \\
\text { voltage (v) }\end{array}$ & $\begin{array}{c}\text { Junction } \\
\text { capacitance }(\mathrm{pF})\end{array}$ & \begin{tabular}{c} 
Resonant frequencies $(\mathrm{GHz})$ \\
\hline 4
\end{tabular} \\
\hline \multirow{2}{*}{0.9} & $1.1,2,2.8,3.51,4.88,5.55,6.3,7$, \\
& & $7.71,8.46$ \\
0.5 & 2 & $1.07,1.97,2.76,3.26,3.51,4.88$, \\
& & $5.66,6.32,7.72,8.49$ \\
\hline
\end{tabular}

allows a fine tuning of frequencies in the spectrum of selective band as function of diode junction capacitance as shown in Figure 11(a). The excited notch resonant frequencies for the spiral with two different diode reverse bias capacitances are illustrated in Table 2. The radiation pattern plots for the notch band mode of antenna are not presented here, since they are comparable to those of antenna operating without any diode biasing. 


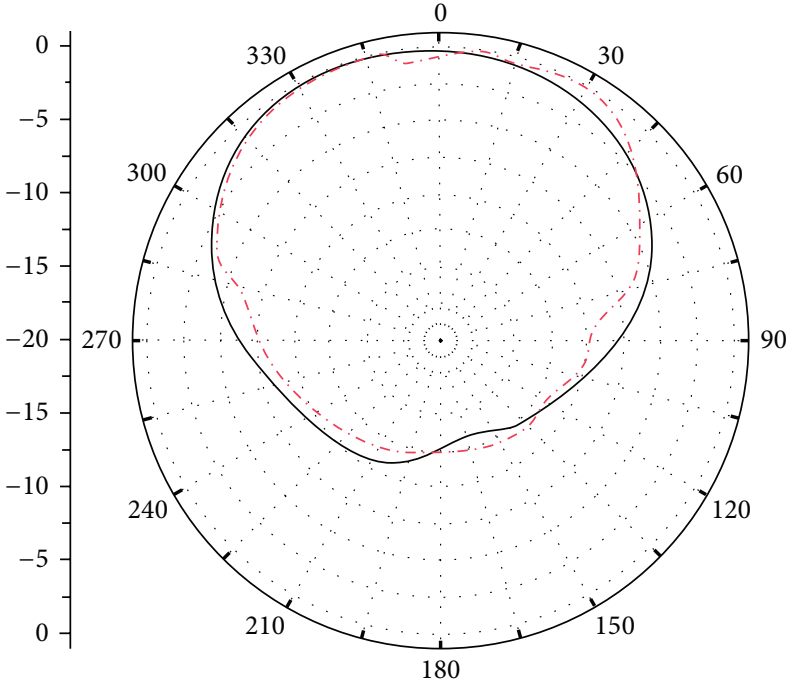

(a) $1.93 \mathrm{GHz}$

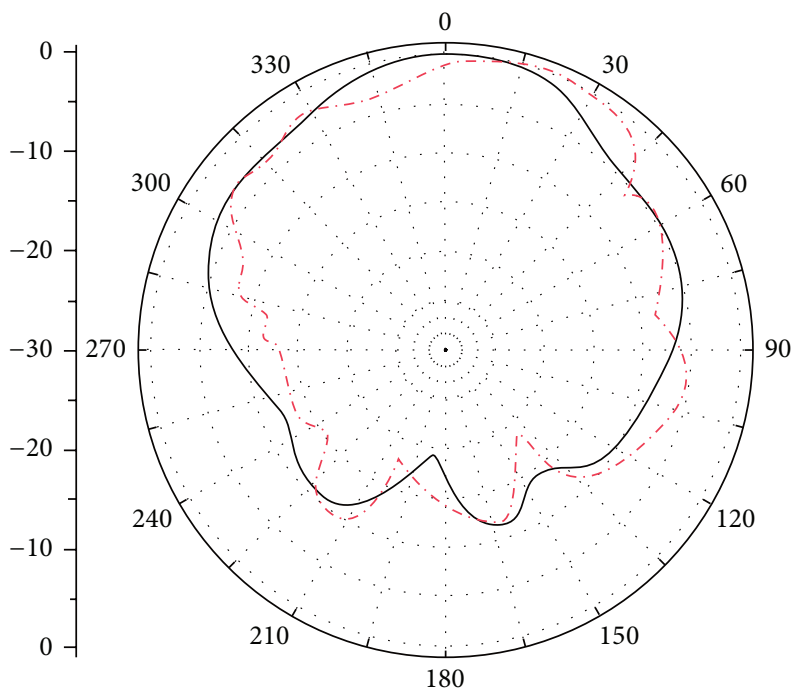

- Simulated

.... Measured

(c) $3.47 \mathrm{GHz}$



(b) $2.79 \mathrm{GHz}$

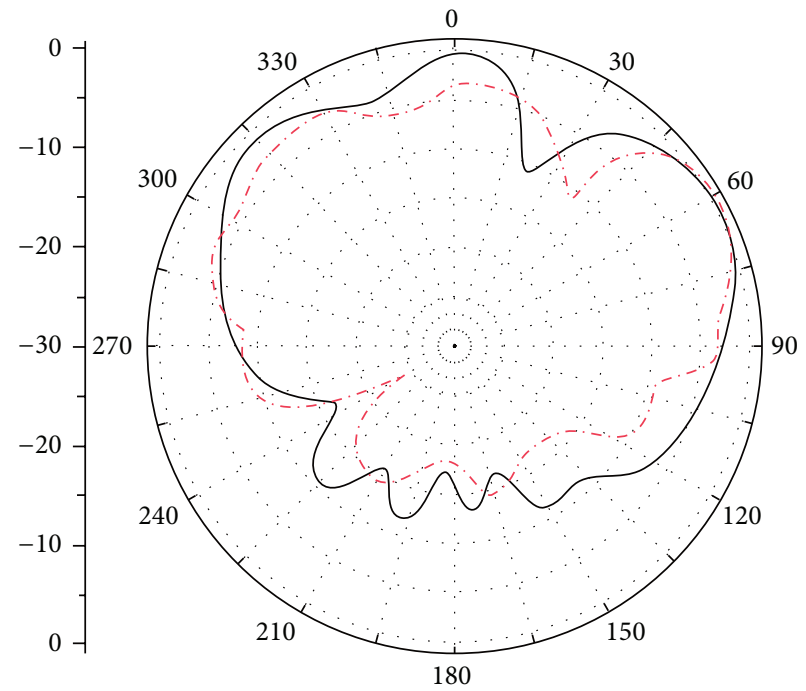

- Simulated

-... Measured

Figure 10: Relative power pattern $(\mathrm{dB})$ plots in E-plane $(x z)$ without diode.

TABLE 3: Resonated frequencies for different diode ON-OFF conditions in ground plane.

\begin{tabular}{lcc}
\hline Diode ON & Diode OFF & $\begin{array}{c}\text { Resonant frequencies }(\mathrm{GHz}) \\
\text { Selective band/wideband }\end{array}$ \\
\hline D1 & D2, D3 & $\{1.89,2.63,3.23,3.66,3.82,4,4.38,4.99,5.28,5.64\} /\{6.15-10\}$ \\
D1, D2 & D3 & $\{1.89,2.38,2.63,3.28,3.42,3.66,3.84,4,4.39,4.98,5.32,5.64\} /\{6.15-10\}$ \\
D1, D2, D3 & - & $\{1.19,1.72,2.15,2.33,2.62,3.26,3.66,3.85,4,4.42,4.97,5.33,5.63\} /\{6.13-10\}$ \\
\hline
\end{tabular}

Lastly, the switching action of three varactor diodes placed in the antenna ground plane provides a frequency band conversion back from multiple notches to notches and wideband which can be seen in Figure 11(b). Three different ON-OFF conditions are used in order to have increased frequency reconfigurability option in the design.
The diode ON-OFF conditions and their corresponding resonant frequencies are illustrated in Table 3. However, here in this case, a variety of radiation pattern are observed in the shape of omnidirectional, butterfly, eight and flower petal. This explains that, with diode placed in the antenna ground plane, both frequency and pattern reconfigurability have been 


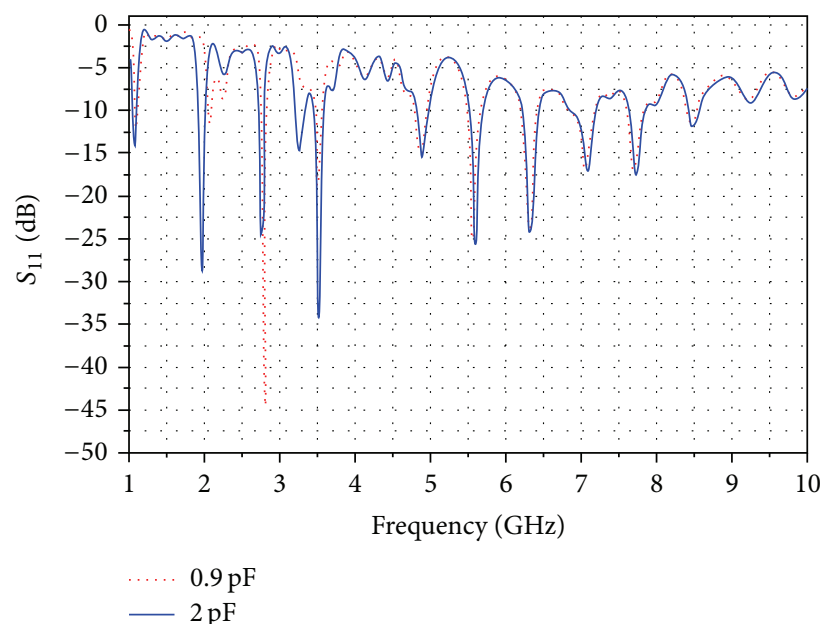

(a)



(b)

FIGURE 11: Computed reflection coefficient plot with (a) diode bias and (b) with ground plane diode switching.



(a)

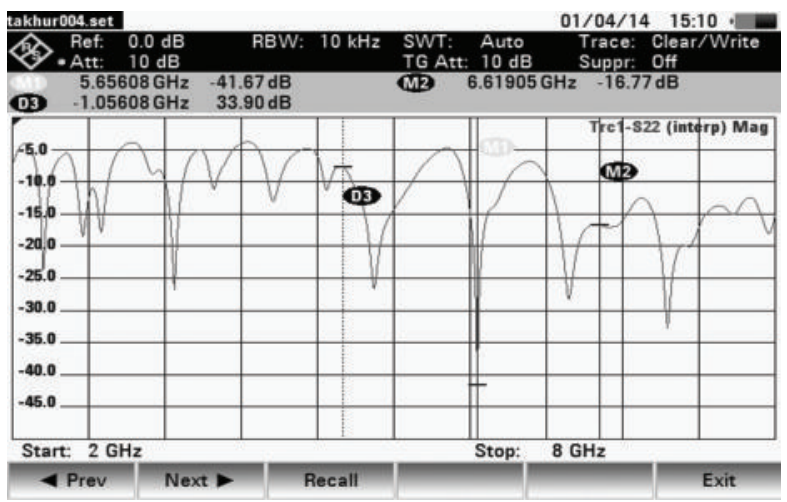

(b)

FIgURE 12: Measured plot of $S_{11}$ (a) at $0.9 \mathrm{pF}$ and (b) at $2 \mathrm{pF}$.

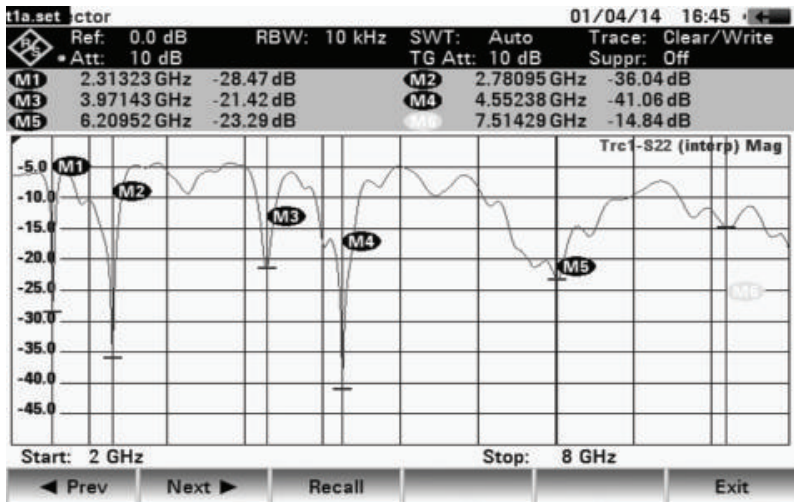

(a)

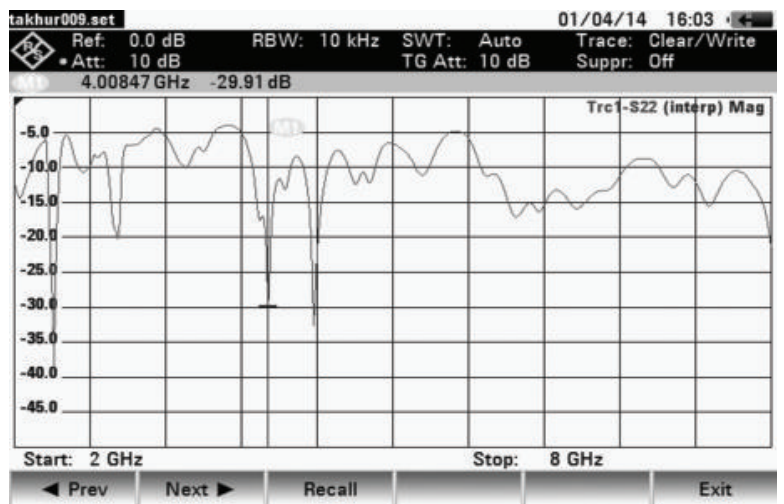

(b)

Figure 13: Measured plot of $S_{11}$ (a) at D1 condition (b) at D1 + D2 condition.

achieved (Figures 12 and 13). The unique pattern plots in Eplane for D1, D1 + D2, and D1 + D2 + D3 condition at some of the resonant frequencies are shown in Figures 14 and 15. The polarization for $\mathrm{D} 1$ condition at $1.89 \mathrm{GHz}$ and $2.63 \mathrm{GHz}$ is vertical and at $4.99 \mathrm{GHz}$ is horizontal, whereas for D1 + D2 + D3 it is again horizontal polarization. However, small grating lobes are observed at $4.98 \mathrm{GHz}, 4.99 \mathrm{GHz}, 5.28 \mathrm{GHz}$, $5.23 \mathrm{GHz}$, and $5.64 \mathrm{GHz}$, because these are the frequencies at which more than one spiral arm has dominant current distribution. 


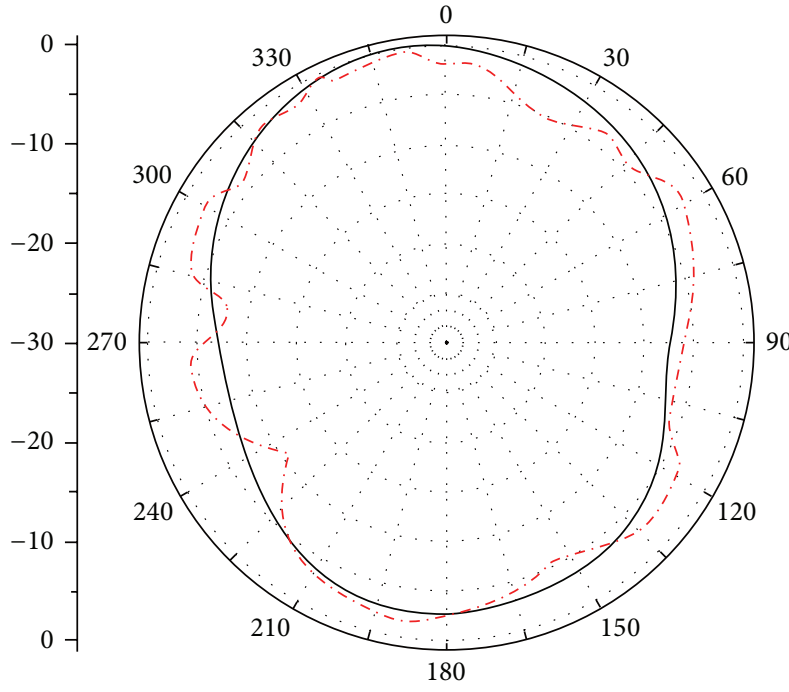

(a) $1.89 \mathrm{GHz}$

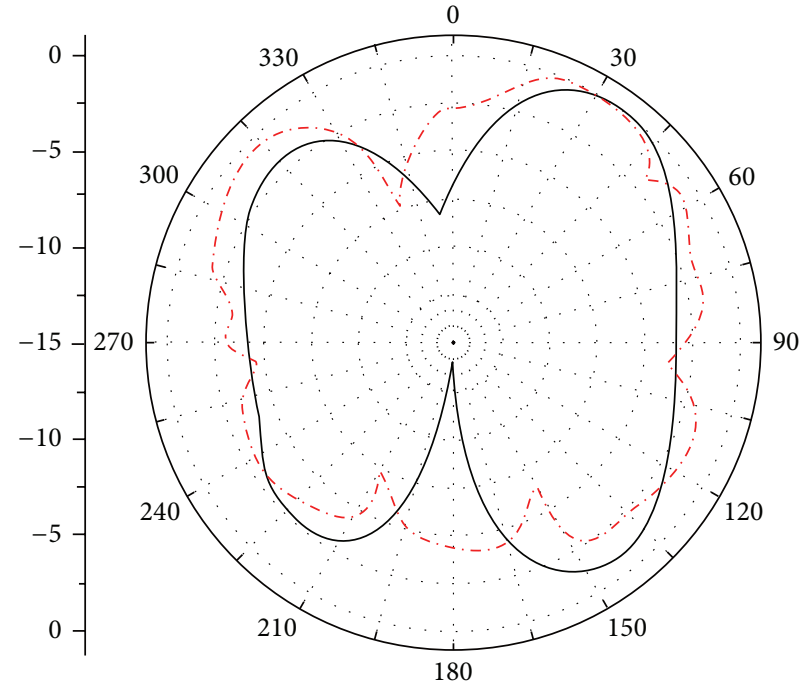

(b) $2.63 \mathrm{GHz}$

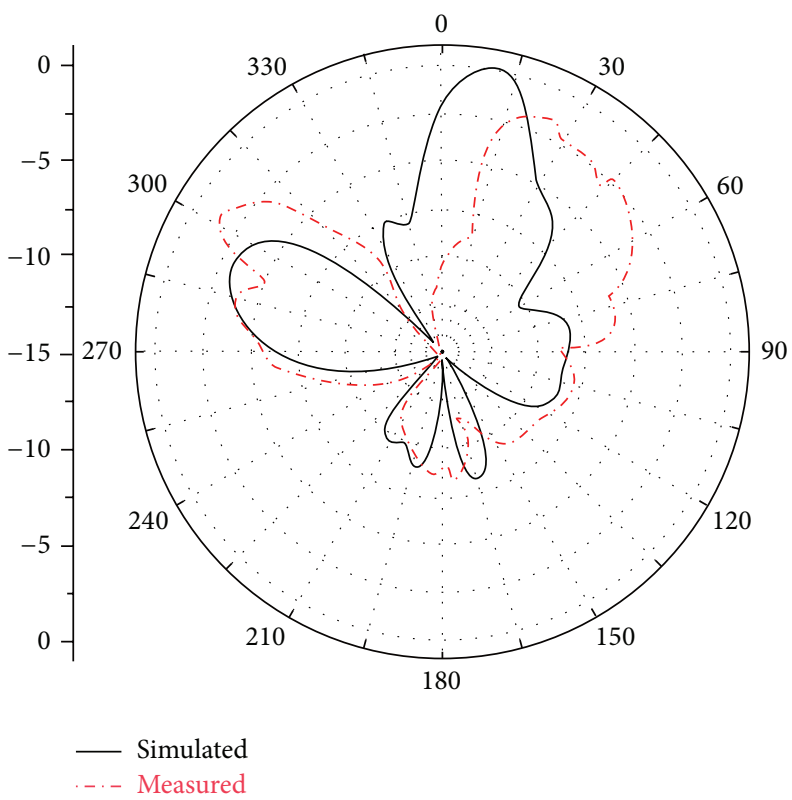

(c) $4.99 \mathrm{GHz}$

FIGURE 14: Relative power pattern $(\mathrm{dB})$ plots in E-plane $(x z)$ for D1-ON.

The radiation pattern plots for $\mathrm{D} 1+\mathrm{D} 2$ configuration are not illustrated since they are comparable to those with D1 configuration.

It has been observed that a slight shift in the measured frequency response has occurred in diode bias conditions and ground plane diode switching conditions. This is possibly due to the rough soldering at the terminal joints of varactor diode and DC blocking capacitor. However, the unique pattern of frequency response as that of simulated results is also preserved in the measured results.

\section{Fabricated Design}

The design shown in Figure 16 is fabricated on lossy FR-4 material and a thin layer of tin has been coated on the antenna to prevent oxidation of copper material. The RF choke and blocking capacitor is of ceramic type with SMD package. Standard $50 \Omega$ SMA connector is used to provide RF feed in the antenna. The red wire indicates a +ve DC connection and black wire is for DC ground.

\section{Conclusion}

A microstrip configured planar reconfigurable spiral antenna design has been demonstrated. The unique frequency response of notch and wideband together makes the antenna suitable to be used for front-end system of cognitive radio. Also the frequency band conversion from notch and wideband to multiple notch frequencies by placing a varactor diode at suitable position along the spiral arms not only 




Figure 15: Relative power pattern (dB) plots in E-plane $(x z)$ at 2.15 GHz for D1, D2, and D3-ON.

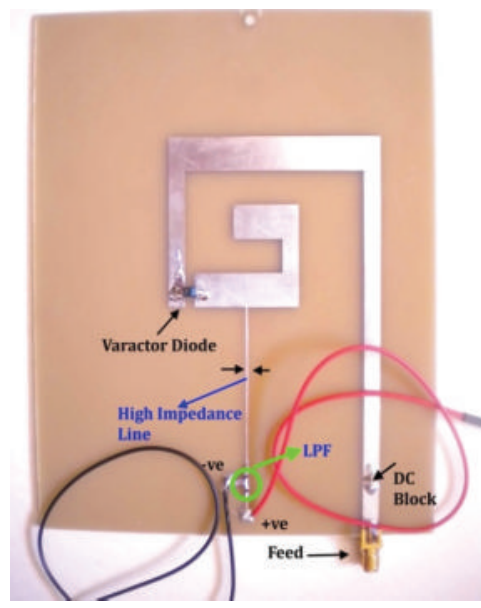

(a)

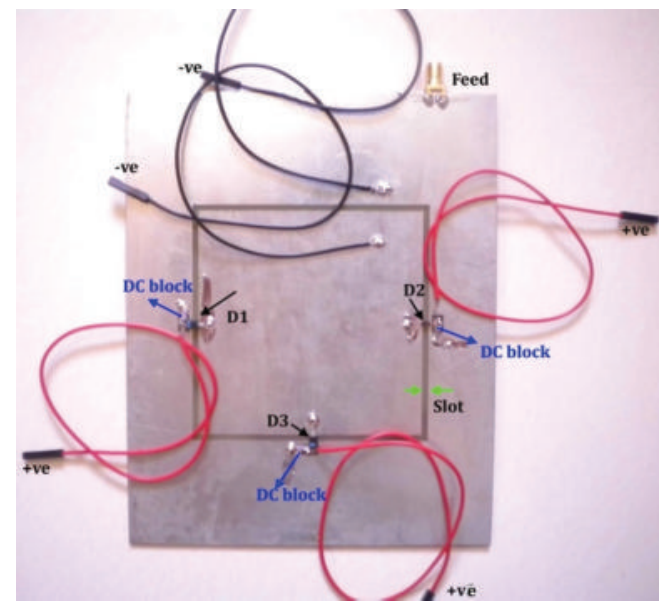

(b)

FIGURE 16: Implemented printed circuit board, (a) antenna view and (b) ground plane view.

increases support of various frequency channels but also helps to avoid adjacent channel interferences due to wideband. The measured and simulated results of the proposed design without any diode biasing are in close agreement to each other. It is found that the radiation patterns at resonant frequencies are almost comparable when the antenna is operating without any diode biasing. Thus we can say that designed antenna exhibits frequency reconfigurability. However, grating lobes which are the undesired lobes do exist when the antenna ground plane is modified with three diode switching conditions.

The future work aims to find techniques to minimize the grating lobes and develop a smart array of such antenna design which will eventually help to provide a greater degree of freedom in frequency scanning of spectrums for the various mode of cognitive radio.

\section{Conflict of Interests}

The author declares that there is no conflict of interests regarding the publication of this paper.

\section{Acknowledgments}

The author would like to thanks Chitra Takle for her support in this work and Don Bosco College of Engineering for providing vector network analyzer for antenna testing. 


\section{References}

[1] P. E. Mayes, "Frequency-independent antennas and broad-band derivatives thereof," Proceedings of the IEEE, vol. 80, no. 1, pp. 103-112, 1992.

[2] J. D. Dyson, “The equiangular spiral antenna," IRE Transaction of Antenna and Propagation, pp. 181-187, 1959.

[3] J. A. Kaiser, “The Archimedean two-wire spiral antenna," IRE Transactions on Antennas and Propagation, vol. 8, no. 3, pp. 312323, 1960.

[4] R. Bawer and J. J. Wolfe, "A Printed Circuit Balun for use with spiral antennas," IEEE Transaction on Microwave Theory and Techniques, vol. 8, no. 3, pp. 319-325, 1960.

[5] V. Trifunović and B. Jokanovic, "Four decade bandwidth uniplanar balun," Electronics Letters, vol. 28, no. 6, pp. 534-535, 1992.

[6] J. Thaysen, K. B. Jakobsen, and J. Appel-Hansen, "A wideband balun-how does it work?" Applied Microwave and Wireless, vol. 12, no. 10, pp. 40-50, 2000.

[7] R. Garg, P. Bhartia, I. Bahl, and A. Ittipiboon, Microstrip Antenna Design Handbook, Artech House, London, UK, 2003.

[8] J. R. James and P. S. Hall, Handbook of Mirostrip Antennas, vol. 1, Peter Peregrinus, London, UK, 1989.

[9] “Computer Simulation Tool,” Microwave Studio Suite, 2010. 

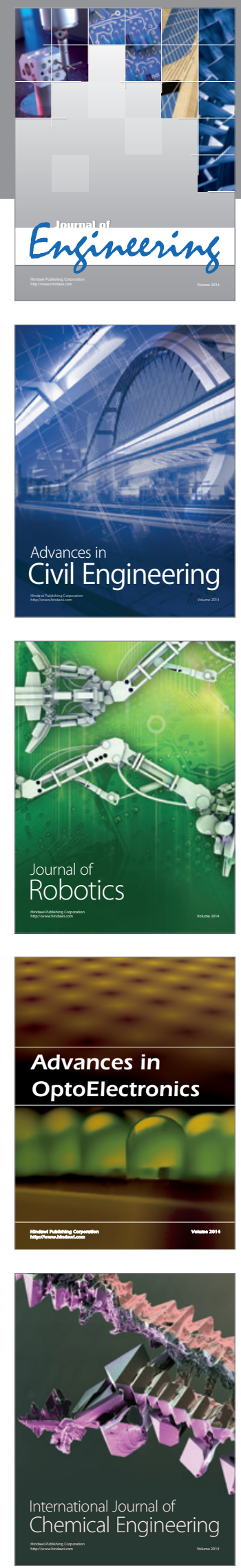

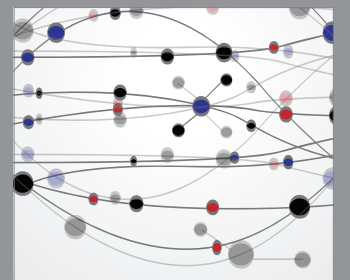

The Scientific World Journal
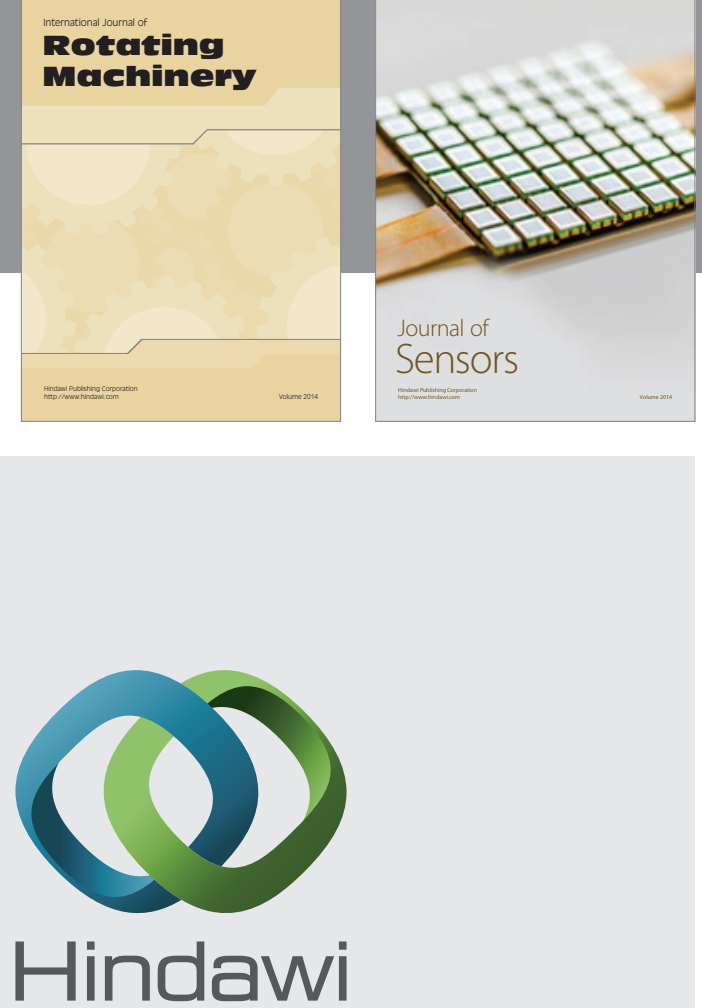

Submit your manuscripts at http://www.hindawi.com
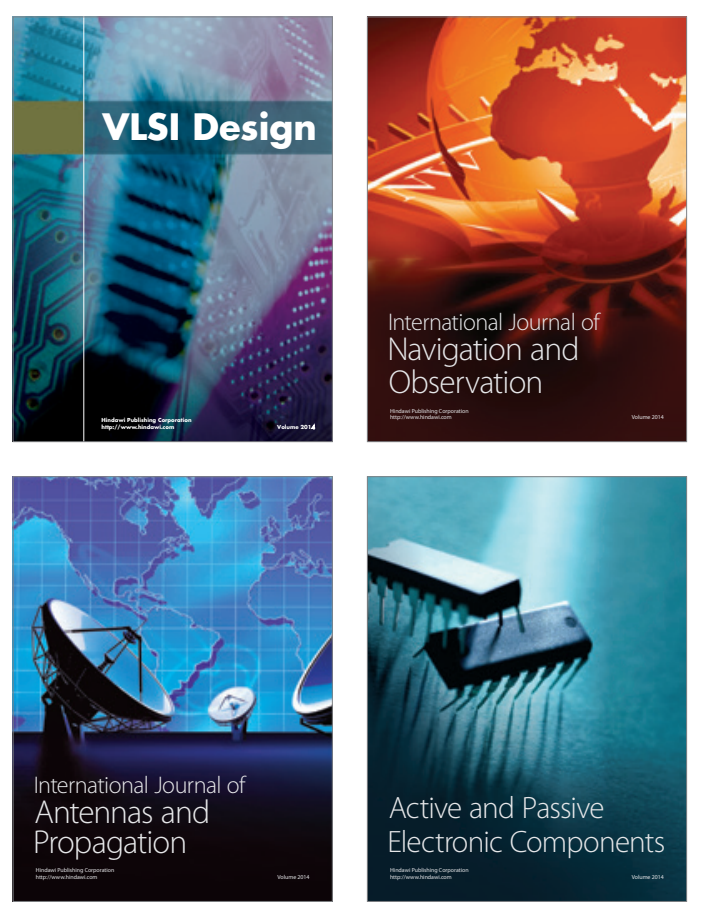
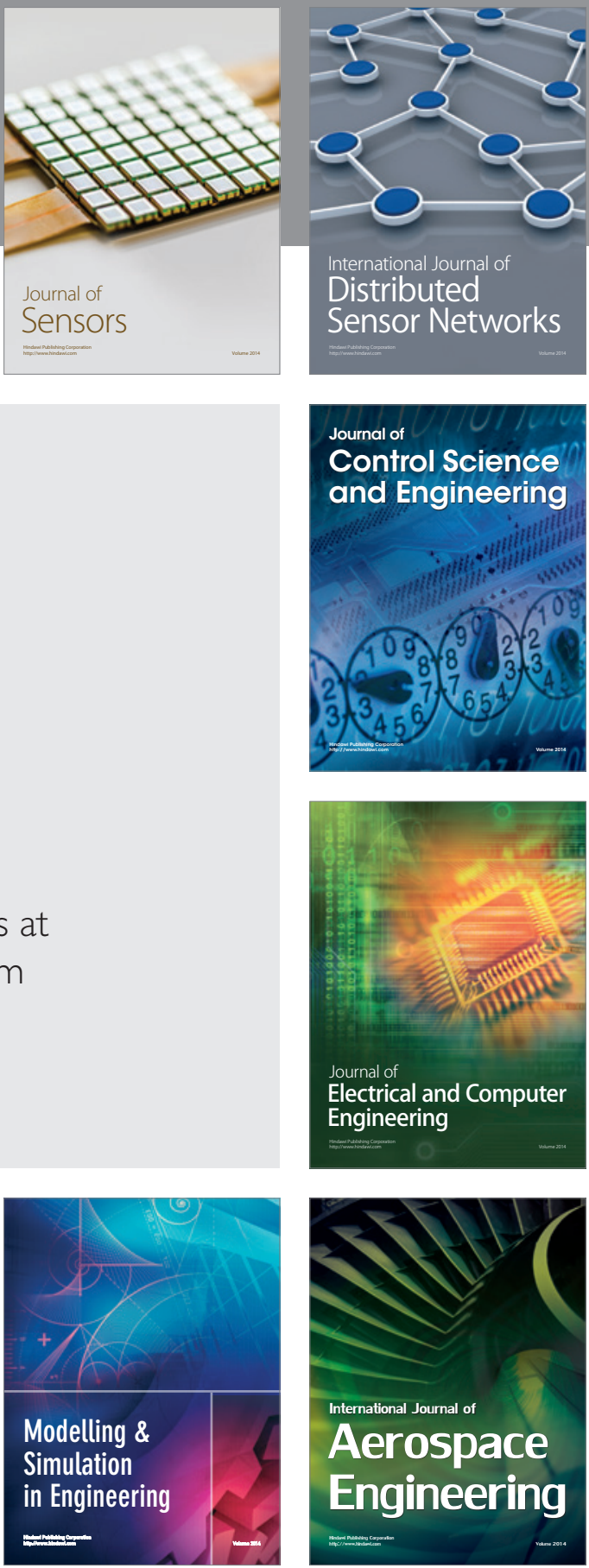

Journal of

Control Science

and Engineering
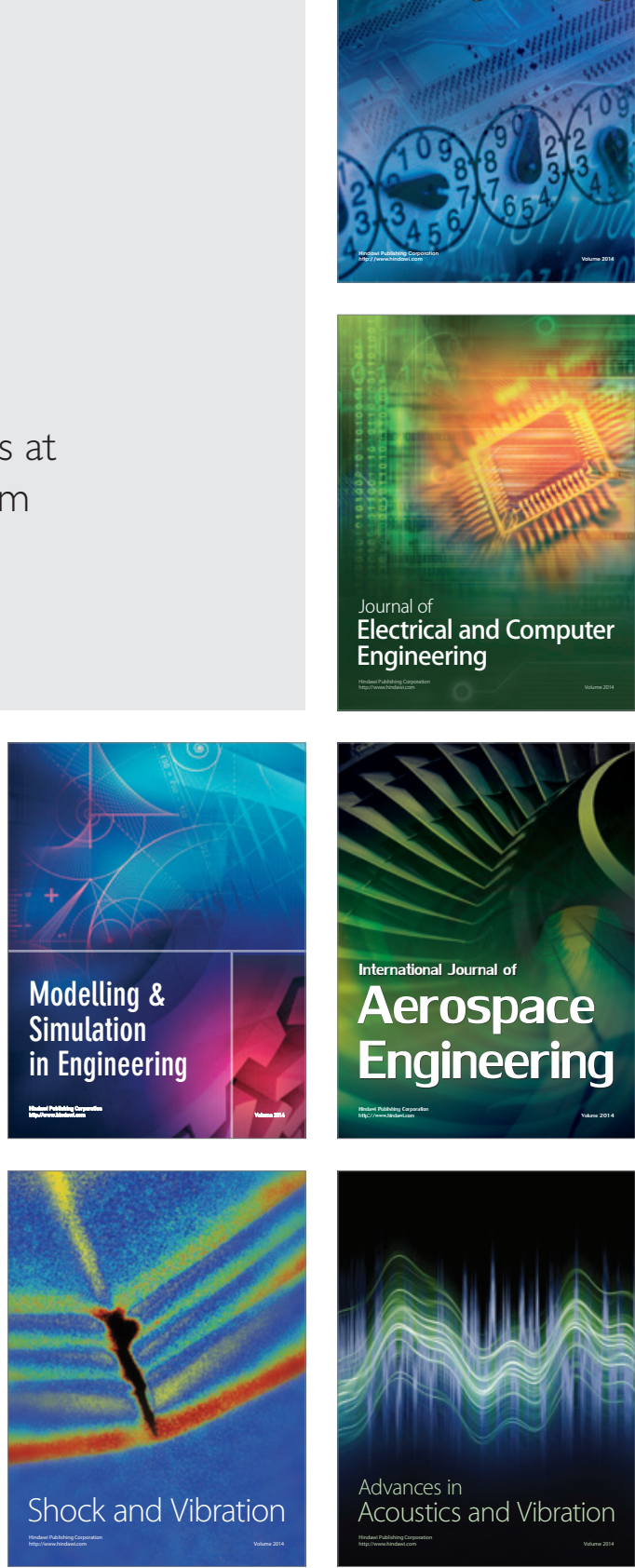